\title{
Pancreatic Cancer Screening: Time for New Markers?
}

\section{Maddalena Zippi}

Unit of Gastroenterology and Digestive Endoscopy, Sandro Pertini Hospital, Rome, Italy

Recent epidemiological data on pancreatic cancer presented at the 24th United European Gastroenterology (UEG) Week held in Vienna (Austria) on October 2016, is alarming. During his lecture, Professor Matthias Löhr (UEG pancreatic cancer expert from the Department of Clinical Science, Intervention and Technology, Karolinska Institutet, Stockholm, Sweden) has said: "Pancreatic cancer survival rate is lower than any other cancer. Consequently, it is absolutely vital that patients receive a diagnosis as early as possible to allow for surgery, which is currently the only potential for a cure." This important statement comes from a recent study in which the authors used time-linear prediction models to show how mortality rates for pancreatic cancer will increase across Europe (EU) by approximately $50 \%$ in 2025 compared to that in 2010 , moving from 76,000 deaths to 111,500 deaths [1]. In addition, the study also revealed that pancreatic cancer may become the third leading cause of death from cancer in the EU following lung and colorectal cancers [1]. We know that the established risk factors are obesity, diabetes, smoking, family history, chronic pancreatitis, and alcohol consumption. These factors should be kept under increased surveillance in those patients having a greater risk of developing pancreatic cancer. Given that these symptoms are not easily identifiable, it is difficult to make early detection of neoplasms so as to avoid recourse to surgery. In fact, at diagnosis, only about $20 \%$ of patients with pancreatic cancer are considered to be eligible for surgical treatment [2]. It is well known that pancreatic ductal adenocarcinoma (PDA) is one of the most difficult types of neoplasia in terms of both diagnosis and treatment. Over the past few years, several serum and tissue markers have been proposed for identifying the earliest forms liable to greater effective treatment, since the prognosis remains low. To date, the most used marker is the carbohydrate antigen (CA 19-9) [3]. According to data from the European Group on Tumor Marker guidelines, this marker is not useful in the diagnosis of early forms [4], utility which instead assumed in concert with radiological techniques, such as computed tomography (CT) or endoscopic ultrasound (EUS). Adnab-9 is a promising murine monoclonal antibody (MAb) that can identify a subpopulation of cells in colonic adenomatous but not carcinomatous tissue resembling Paneth cells $[5,6]$. Adnab-9 MAb raised against neoplastic, potentially premalignant colonic adenomas recognizes a marker in colonic effluent or tissue which correlates with the presence of tumors or risk of colorectal cancer (CRC) [7]. Adnab-9 is shed into the bowel lumen [8], and preliminary data show that it is also detectable in the stool [9]. Tobi et al. [10] have studied whether Adnab-9 may also be a useful prognostic marker for invasive intraductal papillary mucinous neoplasia (IPMN). They found that $26 \%$ of the IPMNs were labeled with Adnab- 9 as compared to $0 \%$ of pancreatic ductal cancers or surrounding benign tissues $(\mathrm{p}<0.001)$. Labeling in IPMNs was usually seen in the noninvasive epithelium, suggesting that Adnab-9 is a premalignant marker in these kinds of lesions [10]. Recently, in a prospective study, Tobi et al. [11] evaluated Adnab-9 monoclonal antibody in stool, pancreaticobiliary secretions, and tissue as screening and prognostic value in sporadic PDA. In particular, pancreaticobiliary secretions were collected from 12 PDA patients and 9 controls. Their results showed that Adnab-9 alone was diagnostic and prognostic when measured in pancreatic secretions, feces, and tissues of PDA patients compared to the controls $(\mathrm{p}<0.05)$. They concluded that Adnab-9 fecal binding can precede the clinical diagnosis by 2.3 years, allowing earlier intervention [11]. In addition,

\section{Publication History:}

Received: January 25, 2017

Accepted: April 25, 2017

Published: April 27, 2017

\section{Keywords:}

Adnab-9, Carbohydrate antigen 19-9 (CA 19-9), Intraductal papillary mucinous neoplasia (IPMN), Pancreatic cancer, Pancreatic ductal adenocarcinoma (PDA)

Mayers et al. [12] have identified a marker in the blood that can help precede the diagnosis of PDA by 2-5 years. They found that elevated plasma levels of branched chain amino acids (BCAAs) (isoleucine, leucine and valine) were associated with a greater than 2-fold increased risk of a future pancreatic cancer diagnosis.

The discovery of new markers is of fundamental importance because it can lead to the early detection of pancreatic cancer. These markers need to be tested on a larger population, and if the data confirms its usefulness, we can begin to effectively counter this tumor.

\section{Conflict of Interests}

The author declare that there is no conflict of interests regarding the publication of this paper.

\section{References}

1. Ferlay J, Partensky C, Bray F (2016) More deaths from pancreatic cancer than breast cancer in the EU by 2017. Acta Oncol 55: 1158-1160.

2. Benson $A B$ (2007) Adjuvant therapy for pancreatic cancer: one small step forward. JAMA 297: 311-313.

3. Duffy MJ, Sturgeon C, Lamerz R, Haglund C, Holubec VL, et al. (2010) Tumor markers in pancreatic cancer: a European Group on Tumor Markers (EGTM) status report. Ann Oncol 21: 441-447.

4. Klapdor R, Aronsson AC, Duffy MJ (1999) Tumor markers in gastrointestinal cancers, EGTM recommendations. Anticancer Res 19: 2785-2820.

5. Tobi M, Maliakkal BJ, Alousi MA, Voruganti V, Shafiuddin M, et al. (1992) Cellular distribution of a colinc adenoma-associated antigen as a defined by monoclonal antibody Adnab-9. Scand J Gastroenterol 27: 737-742.

6. Tobi M, Cats A, Maliakkal BJ, Kinzie JL, Maliakkal R, et al. (1997) Zollinger-Ellison syndrome, acromegaly, and colorectal neoplasia. J Clin Gastroenterol 24: 21-24.

${ }^{*}$ Corresponding Author: Dr. Maddalena Zippi, Unit of Gastronterology and Digestive Endoscopy, Sandro Pertini, Hospital, Via dei Monti Tiburtini 385, 00157, Rome, Italy, E-mail: maddyzip@yahoo.it

Citation: Zippi M (2017) Pancreatic Cancer Screening: Time for New Markers? .Int J Gastroenterol Disord Ther 4: 127. doi: https://doi.org/10.15344/2393$8498 / 2017 / 127$

Copyright: (c) 2017 Zippi. This is an open-access article distributed under the terms of the Creative Commons Attribution License, which permits unrestricted use, distribution, and reproduction in any medium, provided the original author and source are credited. 
Citation: Zippi M (2017) Pancreatic Cancer Screening: Time for New Markers? .Int J Gastroenterol Disord Ther 4: 127. doi: https://doi.org/10.15344/2393$8498 / 2017 / 127$

Page 2 of 2

7. Tobi M, Kaila V, Goo R, (1992) An adenoma-associated antigena as a precolonoscopic marker in familial polyposis coli versus sporadic colonic neoplasia. Gastroenterology 102: A403.

8. Tobi M, Elitsur Y, Moyer MP, Halline A, Deutsch M, et al. (1993) Mucosal origin and shedding of an early colonic tumor marker defined by Adnab-9 monoclonal antibody. Scand JGastroenterol 28: 1025-1034.

9. Yuan M, Xhang X, Liu Y, Tobi M (1996) Sensitivity of fecal Adnab-9 testing for colorectal neoplasia. Gastroenterology 110: A619

10. Tobi M1, Hatfield J, Adsay V, Galagan K, Kozarek R, et al. (2001) Prognostic significance of the labeling of Adnab-9 in pancreatic intraductal papillary mucinous neoplasms. Int J Pancreatol 29: 141-150.

11. Tobi M, Kim M, Weinstein DH, Rambus MA, Hatfield J, et al. (2013) Prospective markers for early diagnosis and prognosis of sporadic pancreatic ductal adenocarcinoma. Dig Dis Sci 58: 744-750.

12. Mayers JR, Wu C, Clish CB, Kraft P, Torrence ME, et al (2014) Elevated circulating branched chain amino acids are an early event in pancreatic adenocarcinoma development. Nat Med 20: 1193-1198. 